\title{
Cardiac involvement in Becker-Kiener muscular dystrophy illustrated by cardiac MRI
}

\author{
Florian Sagmeister ${ }^{1}$, Karin Mild ${ }^{1}$, Thorsten Lieberknecht ${ }^{2}$ and Meinrad Beer ${ }^{1 *}$ \\ ${ }^{1}$ University Hospital Ulm, Department of Diagnostic and Interventional Radiology, Ulm, Germany \\ ${ }^{2}$ Herzklinik Ulm, Dr. Haerer und Partner, Ulm, Germany
}

\section{Introduction}

Becker-Kiener muscular dystrophy (BMD) is as Duchenne dystrophy a X-linked inherited disorder of cardiac and skeletal muscle cells [1]. Cardiac involvement is frequent, mostly unrecognized and yet associated with a high morbidity and mortality [2]. Cardiac MRI (CMR) allows a non-invasive comprehensive analysis of cardiac morphology, function and tissue integrity, the later by late gadolinium enhancement (LGE) [3]. LGE imaging might detect already subclinical cardiac alterations prior to clinical symptoms or echocardiographic findings [4].

\section{Case report}

We report about a 39-year male patient with known BMD, who suffered from dyspnea (NYHA II-III), chest pain and arrhythmias. Echocardiography demonstrated a reduced left ventricular (LV) function and a moderate dilatation of the LV. CMR was performed on a 3-Tesla MRI-scanner (Skyra, Siemens, Germany). Axial T2 haste black blood - sequences of the whole thorax, short and long axis CINE and LGE SSFP - sequences were acquired. Additionally, T1 mapping (Molli) was performed in representative short and long axis views.

Global LV function was reduced to $37 \%$ (Figure 1), the LV was dilated (LVEDVI $106 \mathrm{ml} / \mathrm{m}^{2}$ ). LGE showed a predominantly intramyocardial patchy pattern affecting most basal and midventricular septal and inferolateral wall segments (Figure 2). Region-of-interest (ROI) analysis of post-contrast long axis T1-mapping images detected inhomogenously reduced T1 SI values in septal and lateral myocardium (Figure 3), most pronounced in LGE positive areas of the septum.

\section{Discussion}

Cardiac involvement is life-limiting in BMD. Varghese and Pennell were the first to describe extensive mid-myocardial late gadolinium
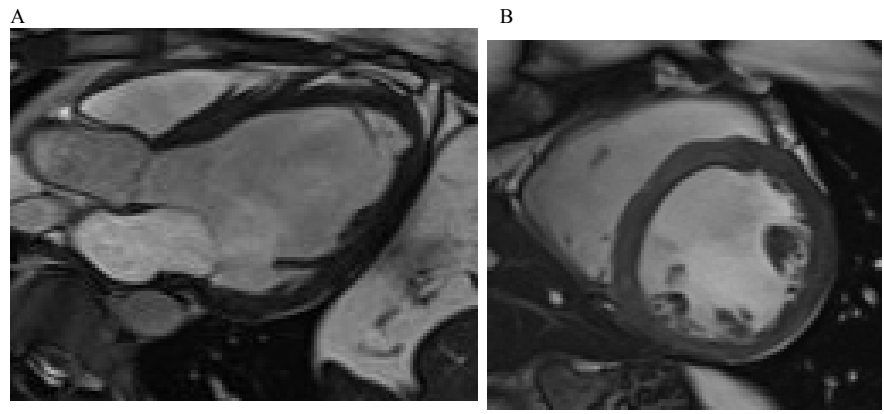

Figure 1. The depiction of left ventricular dilation was possible using long- (1A, 3-chamberview) and short axis (1B) CINE-sequences. enhancement as a hallmark of cardiac disease in BMD [3]. There have also been reports of subendocardial LGE patterns, alluding to the occurrence of myocardial infarctions in these patients [5]. Demonstration of LGE might be a surrogate marker for an impaired clinical outcome [6]. In a series of 15 patients with BMD, Yilmaz and Sechtem showed that CMR with LGE is more sensitive than echocardiography for detection of cardiac alterations [4]. An intramyocardial pattern of LGE positive areas was also seen in the patient presented here. In accordance to the previous reports, mostly septal and inferolateral segments of the LV were affected. Interestingly, also a subepicardial pattern was observed in lateral midventricular parts of the LV, resembling LGE pattern previously reported for myocarditis. A recent large meta-analysis of LGE patterns in non-ischemic cardiomyopathies (NICM), encompassing 9 studies with a total of 1488 patients, proved the negative predictive value for LGE concerning heart failure hospitalization and mortality [7]. Prognostic information of LGE imaging is even increased, when LGE data are quantified [8]. However, the mostly applied methods for
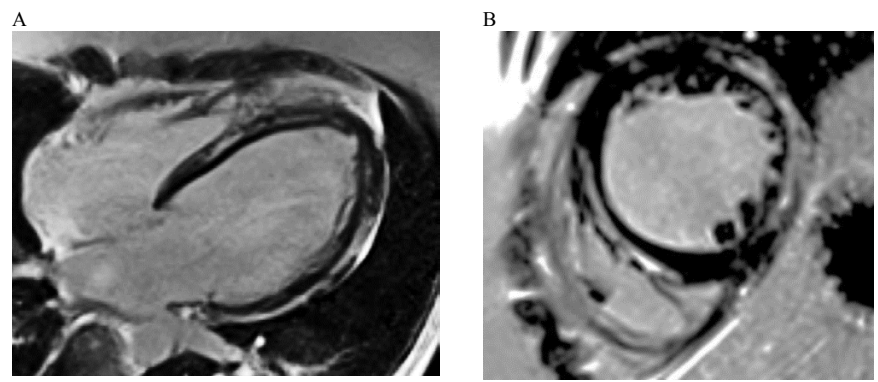

Figure 2. Intramoycardial late gadolinium enhancement (LGE) in septal and inferolateral LV segements. The long-axis view $(2 \mathrm{~A})$ demonstrates the predominantly patchy pattern of LGE areas, the short-axis view (2B) a more confluent pattern, including also the subepicardium.

Correspondence to: Meinrad Beer, University Hospital Ulm, Department of Diagnostic and Interventional Radiology, Albert-Einstein-Allee 23, D-89081 Ulm, Germany, Tel: +49 / 731 / 500-61001; Fax: +49 / 731 / 500-61002; E-mail: meinrad.beer@uniklinik-ulm.de

Special Issue: Imaging of myocardial diseases: to new horizons

Valentin E. Sinitsyn

Professor

Department of Radiology

Federal Center of Medicine and Rehabilitation

Russia

E-mail: vsini@mail.ru

Published: October 18, 2016 


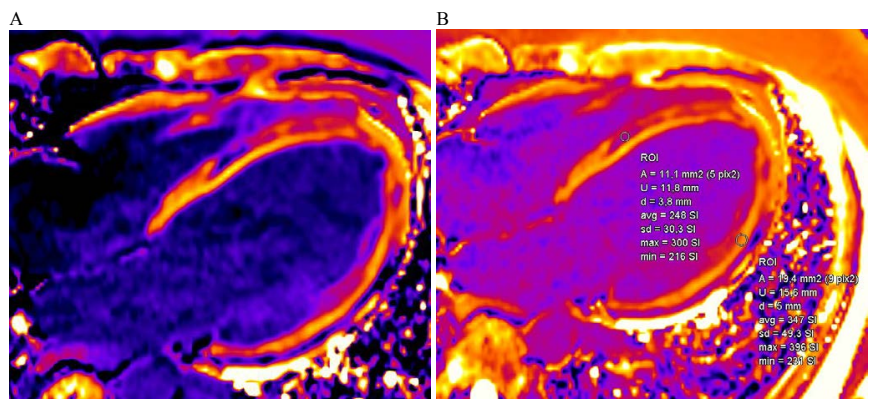

Figure 3. Post-contrast $t 1$ maps in the long-axis view ( $3 \mathrm{~A}$ and $\mathrm{B}$ )- $3 \mathrm{~B}$ depicts the ROI analysis in septal and lateral parts of the LV (SI septal 248. SI lateral 347).

planimetry of LGE areas are time demanding and restricted by intraand interindividual variabilities.

The new method of calculation of tissue T1 values in pre- and/ or post-contrast images might be a more robust and simpler way to establish quantitative data on tissue integrity including the detection of diffuse myocardial fibrosis. Data on $\mathrm{t} 1$ mapping in myotonic muscular dystrophy (DM) type 1 and 2 were the first applications of this technique in muscular dystrophies [9]. We saw a similar reduction of post-contrast $\mathrm{T} 1$ time in the BMD patient presented in this case report. This likely reflects the presence of diffuse myocardial fibrosis, as we saw also a reduction of T1 time in myocardial segments without LGE.

Florian and Yilmaz used a highly sophisticated approach to calculate $\mathrm{t} 1$ mapping results in $\mathrm{BMD}$, generating $\mathrm{R} 1$ maps by taking the reciprocal of each $\mathrm{T} 1$ map on a pixel-by-pixel basis and $\Delta \mathrm{R} 1$ maps were computed by subtracting the pre-contrast R1 map from the postcontrast R1 map [10]. Compared to that, we used a simple visual and semiquantitative ROI approach, leading to the same results. In our opinion, ROI analysis is a robust and easy technique to assess changes in myocardial texture in muscular dystrophy.

\section{Conclusion}

This case reports illustrates the feasibility of a comprehensive analysis of cardiac involvement in BMD by cardiac MRI (CMR). The addition of $\mathrm{t} 1$ mapping opens the possibility to assess diffuse myocardial damage/fibrosis using the robust and simple approach of myocardial ROI-analysis. The reduction of $\mathrm{T} 1$ time is typical in $\mathrm{BMD}$, affecting segments with and without overt fibrosis. In the future, CMR could be applied even in BMD carriers to detect subclinical cardiac involvement like in Duchenne dystrophy [11].

\section{References}

1. Kunkel LM, Hejtmancik JF, Caskey CTh (1986) Analysis of deletions in DNA from patients with Becker and Duchenne muscular dystrophy. Nature 322: 73-7. [Crossref]

2. Melacini P, Fanin M, Danieli GA, Villanova C, Martinello F, et al. (1996) Myocardial involvement is very frequent among patients affected with subclinical Becker's muscular dystrophy. Circulation 94: 3168-75. [Crossref]

3. Varghese A, Pennell DJ (2004) Late gadolinium enhanced cardiovascular magnetic resonance in Becker muscular dystrophy. Heart 90: e59. [Crossref]

4. Yilmaz A, Gdynia HJ, Baccouche H, Mahrholdt H, Meinhardt G, et al. (2008) Cardiac involvement in patients with Becker muscular dystrophy: new diagnostic and pathophysiological insights by a CMR approach. J Cardiovasc Magn Reson 10: 50. [Crossref]

5. Petrie CJ, Mark PB, Dargie HJ (2005) Cardiomyopathy in Becker muscular dystrophydoes regional fibrosis mimic infarction? J Cardiovasc Magn Reson 7: 823-5. [Crossref]

6. Süselbeck T, Haghi D, Neff W, Borggrefe M, Papavassiliu T (2005) Midwall myocardial fibrosis in Becker-Kiener muscular dystrophy. Z Kardiol 94: 465-8. [Crossref]

7. Kuruvilla S, Adenaw N, Katwal AB, Lipinski MJ, Kramer CM, et al. (2014) Late gadolinium enhancement on cardiac magnetic resonance predicts adverse cardiovascular outcomes in nonischemic cardiomyopathy: a systematic review and meta-analysis. Circ Cardiovasc Imaging 7: 250-8. [Crossref]

8. Pöyhönen P, Kivistö S, Holmström M, Hänninen H (2014) Quantifying late gadolinium enhancement on CMR provides additional prognostic information in early riskstratification of nonischemic cardiomyopathy: a cohort study. BMC Cardiovasc Disord 14: 110. [Crossref]

9. Turkbey EB, Gai N, Lima JA, van der Geest RJ, Wagner KR, et al. (2012) Assessmen of cardiac involvement in myotonic muscular dystrophy by T1 mapping on magnetic resonance imaging. Heart Rhythm 9: 1691-7. [Crossref]

10. Florian A, Ludwig A, Rösch S, Yildiz H, Sechtem U, et al. (2014) Myocardial fibrosis imaging based on T1-mapping and extracellular volume fraction (ECV) measurement in muscular dystrophy patients: diagnostic value compared with conventional late gadolinium enhancement (LGE) imaging. Eur Heart J Cardiovasc Imaging 15: $1004-$ 12. [Crossref]

11. Wexberg P, Avanzini M, Mascherbauer J, Pfaffenberger S, Freudenthaler B, et al. (2016) Myocardial late gadolinium enhancement is associated with clinical presentation in Duchenne muscular dystrophy carriers. J Cardiovasc Magn Reson 18: 61. [Crossref]

Copyright: $@ 2016$ Sagmeister F. This is an open-access article distributed under the terms of the Creative Commons Attribution License, which permits unrestricted use, distribution, and reproduction in any medium, provided the original author and source are credited. 